\title{
Frontières
}

\section{Corrida pour soi seul}

\section{Michaël La Chance}

Volume 14, numéro 1, automne 2001

Où est la marge?

URI : https://id.erudit.org/iderudit/1074163ar

DOI : https://doi.org/10.7202/1074163ar

Aller au sommaire du numéro

Éditeur(s)

Université du Québec à Montréal

ISSN

1180-3479 (imprimé)

1916-0976 (numérique)

Découvrir la revue

Citer ce document

La Chance, M. (2001). Corrida pour soi seul. Frontières, 14(1), 59-62.

https://doi.org/10.7202/1074163ar

Ce document est protégé par la loi sur le droit d'auteur. L'utilisation des services d'Érudit (y compris la reproduction) est assujettie à sa politique d'utilisation que vous pouvez consulter en ligne.

https://apropos.erudit.org/fr/usagers/politique-dutilisation/
Cet article est diffusé et préservé par Érudit.

Érudit est un consortium interuniversitaire sans but lucratif composé de l’Université de Montréal, l'Université Laval et l'Université du Québec à Montréal. Il a pour mission la promotion et la valorisation de la recherche. https://www.erudit.org/fr/ 


\section{CORRIDA POUR SOI SEUL}

\section{À JACQUES SANCHIZ}

\section{- INSTANCE MORALE INSISTANCE DE " MOI ॥ - FUGUE MATADOR - PENSER SA VIE C'EST LA DÉTRUIRE}

\author{
Michaël La Chance, Ph.D., \\ professeur d'histoire et de théorie de l'art \\ à l'Université du Québec à Chicoutimi.
}

Ce texte fait suite à la visite que m'a rendue Jacques en novembre 1989. Il se sentait trop mal et savait que les choses ne pourraient continuer comme ça.

Il est comme un poing dont sortiraient des larmes. Il voit la vie le quitter avec désespoir, parce qu'il aime la vie, il aime les femmes, il aime la peinture - et il enrage contre tout le reste. D'une rage qui ne ressemble qu'à lui, à laquelle il abreuve sa fierté, avec la crainte d'être diminué par ses amertumes. Il est homme à préférer une dernière fierté à une longue mascarade.

Lorsqu'il accusait ceci ou cela, j'accusais à mon tour l'alcool et les poudres blanches. Si j'avais su ce qui allait suivre, je ne lui aurais sans doute pas assené un galimatias sur la nécessité de vivre - j'aurais été tout entier au bonheur de retrouver l'ami, quelqu'un qui n'a rien que ce qu'il est, d'une vérité que ne diminue en rien son désarroi. Mais les choses se sont passées autrement.

Un combat s'est engagé entre nous, où je cherchais à préserver la vie de sa mort, d'autant qu'il avait déjà quelque peu versé de l'autre côté. Maintenant que c'est fini, je veux parler de lui, comme d'un aficion de la vie très particulier.

il falloit qu'il considérât son état devenu intolérable, qu'il falloit en sortir par quelque voie que ce fût et que toute voie lui étoit fermée hors celle que je lui avois présentée; qu'elle étoit dure, cruelle, mais unique; qu'après tout, il falloit bien qu'il se séparât un jour de celle qui le tenoit sous son joug; qu'un engagement si long, si éclatant l'avoit précipité dans un abîme sans fonds ; que le jour de s'en arracher étoit venu, et qu'il ne tenoit qu'à lui de se faire de cet abîme un degré d'honneur...

Louis... duc de Saint-Simon

\section{I}

L'ornière devient plus profonde, il n'est plus qu'une tête qui court sur la crête d'un sillon, tête dure comme un soc brillant sur les deux faces. Engouffré dans son destin, il appelle ce qui est distant, pour parvenir à cette distance si prononcée qu'il n'est plus parmi nous. Il appelle ce qui est différent en lui-même pour parvenir à cette différence dans laquelle il se découvre ailleurs. La solitude le ronge, est devenue une plus grande souffrance - bientôt il semblait à portée, si proche qu'il suffit d'étendre la main pour le trouver là, accroupi, la tête dans les épaules, avec une obscurité dans ses yeux que ne sauraient dissiper ses paupières.

Si proche! Certains ont eu peur lorsqu'il leur a tendu la main!

Comment s'empêcher d'aller à la mort lorsqu'elle est, depuis si longtemps, par la souffrance, une alliée délétère?

À ce point ce n'est plus un homme qui met fin à ses jours. L'Ombre, après avoir tout ravagé, finit par s'attaquer elle-même. C'est une ombre brutale, dont les sabots nous martèlent la tête surchauffée de soleil - avec le glas de l'enclume.

L'Ombre revient, le frôle. Il s'est dressé, il se tient le plus droit afin que rien ne déborde, surtout pas le désordre de sa vie, où l'Ombre pourrait l'encorner. Cogida.

Il n'a plus de ventre, il le tient dans ses mains. Son enfance lui avait laissé une plaie au flanc, il a passé cette vie plié sur luimême - seule la poudre lui fait oublier sa rage. Les lendemains lui laissaient les mains trop pleines de lui-même.

Il a vu l'Ombre s'étendre, il a su qu'elle ne tarderait pas à l'engloutir.

Le ciel matinal garde les traces du passage de la nuit, l'obscurité lui revient par des nausées crépusculaires qui voûtent la fin du jour.

La pénombre revient trop rapidement, se soulève dans les choses devenues menaçantes.

La solitude l'a privé de ses appuis matériels, il ne peut faire un geste de plus. Le matin revenu, il ne peut plus marcher dans la rue sans avaler ses larmes. Il a pour dernier lien avec le monde, pour seul pas de l'avant cette souffrance renouvelée, toute comprimée. Peut-être lui aurait-il fallu infliger cette souffrance à d'autres, ce qui est parfois un moyen d'exister, tant d'autres existent par cette crispation. Il s'y refusait. Elle était trop grande pour la patience des mots, il est maintenant trop tard pour commencer à la dire, quand tout viendrait en même temps. Il continue à exister par les moyens qu'il improvise.

\section{II}

Le rire se met à table: la douleur est fille de l'absence et l'absence le trou que s'évertue à boucher le spectacle du monde...

Alain Monvoisin

Il cède à lui-même. Il multiplie les lâchetés pour retourner à cette vérité trop sèche, trop froide: nous ne valons guère plus que le néant dont nous avons déjà pris le détour, puisque c'est depuis ce néant que nous nous regardons vivre. Qu'avons-nous fait? Nous l'avons harcelé de tous côtés, désireux de le restituer au plaisir de vivre, alors qu'il sombrait dans sa meurtrissure - peut-être avonsnous cru que la mort s'étend comme un mauvais rêve, peut-être avons-nous cru que c'est un rêve que l'on peut dissiper.

Alors je l'ai accompagné un bout du chemin, sans me demander s'il y aura quelqu'un pour le rattraper, si je n'aurais pas été celui qui l'aura définitivement lâché, celui qui au plus creux l'aura abandonné.

Ce dernier geste - certains ont simplement relâché la main, d'autres lui ont demandé de partir - me paraît infêmence, comme si je l'avais poussé du haut d'une falaise. Mais ce gouffre, il l'aura creusé. Il y tourne depuis longtemps, en spires plus resserrées qu'il nous avait paru, lorsque nous l'avions accompagné quelques tours.

Il était déjà au fond quand nous l'avons lâché, mais la chute fut terrible - comme s'il avait rattrapé tout d'un coup une verticalité qu'il avait ramassée depuis longtemps. Ce dernier geste, d'ouvrir la main par laquelle il tient à la vie, la main ouverte comme un signe d'adieu, est la pierre jetée dans le puits pour en brouiller le disque d'argent. 
Si grande est sa solitude qu'il ne tient à la vie que par la main ouverte, dans un signe d'adieu à l'ami lointain.

Une telle meurtrissure apparaît obscène, il semble qu'on ne saurait la dire. Autant dire comment la pensée se perd lorsqu'elle croit se comprendre elle-même. Déperdition impensable puisque l'homme a choisi de dire la mort par un suspens de sens, ne cesse depuis de faire sens pour substituer cette transparence à une autre, plus glacée.

Une corrida pour soi seul, c'est imaginer toutes les morts possibles, tous les jeux de cape devant l'Ombre. C'est prévoir les variations de la souffrance, c'est imaginer à chaque fois le retour de la souffrance en quelque région lointaine de soi-même. On la prévoit en effet pour l'y attirer et s'en préserver quelque peu.

En cette heure de détresse il n'est de nudité assez grande. Cet homme si fier nous implore de ne pas avoir honte de lui. Il se laisse éblouir par un ciel vide.

Il perd ses assises dans le quotidien, il n'offre plus de résistance au temps de grande altitude qui l'emporte.

L'esprit, entité nébuleuse et flottante, entre en communication avec les corps et l'espace qu'ils occupent par une infinité de fibres très sensibles. La brûlure des abus a pour effet d'isoler l'esprit, de le laisser à luimême. Une longue insomnie de jour comme de nuit recouvre la réalité d'un voile douloureux. Cet homme, jadis si réservé, n'entrevoit plus la réalité que par la déchirure de son âme, c'est le monde qui s'éloigne dans une accélération insensée.

La vie, devenue une ivresse, le laisse encore plus seul et abandonné après chaque débauche de bonheur. Et lorsque ce n'est pas assez, il lui faut transformer l'espoir en vertige, se vider de ses espérances, se libérer de tout regret de l'avenir et assassiner ses souvenirs. Là-bas, dans la ville, il lui faut maintenant se priver de ses années de soleil - il avait longtemps couru dans la colline avec pour bagage d'enfant une tranche de pain arrosée d'un peu d'huile d'olive.

J'essaie d'imaginer sa dernière journée de bonheur, de rieuse insouciance. Il avait sa fierté, un dard douloureux, fiché à travers lui, qui l'empêchait de plier et l'a fait mourir trop longtemps debout.

Paris qu'il ne pouvait plus quitter. Il y a des lieux qui nous font vivre juste assez afin que nous recevions d'eux notre mort.

\section{III}

La corrida relève du Sacré parce qu'il y a menace de mort et mort réelle, mais ressentie, éprouvée par d'autres, avec d'autres.

Imaginez une corrida pour vous tout seul. (à expliquer longuement)

Laure Bataille
Dans sa déperdition de vie, il est plus vivant que nous tous - c'est son habit de lumière, son mépris qu'elle ne soit rien que cela. La vie se venge de tout ce qui a plus d'orgueil qu'elle. Il est prêt à la combattre dans l'arène, pour un combat qu'elle ne saura gagner - car c'est la vie même qui coule dans le sable avec le coup de corne qu'elle lui donne.

L'un reste spectateur, l'autre, enfoncé dans la meurtrière, se sent observé dans ses moindres gestes. C'est ainsi que de geste en geste, quelques-uns se sont avancés et rejouent une signification qu'ils ne connaissent pas. Quelques-uns éprouvent une terreur sacrée à la contemplation des abandons et de la résistance acharnée que peut offrir l'autre d'eux-mêmes, lorsque l'autre attise son angoisse à la corne de l'Ombre.

Comment assister au viol de l'âme, sans croire que l'Ombre sera terrassée? Elle s'élance depuis l'obscurité du chiquero, traîne avec elle la pénombre du théâtre. Le sable brûlant dans les yeux, elle s'élance tête baissée vers ce qu'elle accuse. Le temps de lire les signes dans l'étoffe de la muleta, il est trop tard, la fatigue est déjà trop grande, il ne reste plus que la rage d'avoir été joué par sa propre vie.

La cape fait claquer la lumière, pour exciter une fulgurance dans le regard. La bête s'élance, elle court sauvagement dans un monde de grisailles qu'éclaire notre vie encore plus lourde de pénombre.

Folie du torero qui affronterait l'Ombre sans le soutien des spectateurs. Folie d'engager une corrida pour soi seul.

\section{IV}

Corrida pour soi seul - on cherche à se rejoindre par une contorsion dont on ne revient pas, même si on ne s'y rejoint pas, qui devient cette impasse à soi, une impasse si étroite que nous ne pouvons plus y loger.

Jusqu'où faut-il pousser l'humilité avant d'accepter de mettre la vie dans une arène, de la mettre en jeu dans le cercle de notre conscience? La vie porte en elle une intensité qui la tue: nous ne pouvons vivre qu'à puiser notre énergie dans cet exercice si cruel, dans un sursaut qui nous consume. Desplante.

Jusqu'où faut-il pousser la tromperie, pour paraître si droit, aujourd'hui ? Le désir de vérité produit l'aplanissement de tout - par la tromperie. Alors comment recommencer, quand on pourrait, par un doigt dressé - un signe qui s'efface - indiquer le point zéro d'où les choses redeviennent possibles. Nous sommes ces éreintés pour lesquels la vie est un vide à remplir par l'agitation la plus vaine, qui ont des intérêts parce que c'est l'anse par laquelle ils saisissent ce vide.

Corrida pour soi seul. Nous apprivoisons la mort en faisant d'elle un atout. Notre mort saura provoquer le remords de n'avoir pas été plus généreux, le regret de n'avoir pas été plus juste. C'est pourquoi on tient sa mort en réserve, comme un acte de justice. Lorsque cette dernière carte tombera sur la table, personne n'aura le souci de la regarder.

Lorsque notre vie nous échappe, nous croyons en mieux retenir le mystère de le confier, par bribes, à nos intimes. Il semble que nous pouvons donner à d'autres la part de nous-même que nous possédons le moins. Alors nous portons la gravité de la mort comme une haute idée de la vie.

- Comment flotter avec nos souffrances passées, que réveillent nos souffrances présentes - comment flotter dans une dérive sans repères et nommer notre sillage le temps?

- Comment prendre l'habitude des inégalités qui mettent certains hommes en dessous des autres? C'est un pont d'obscurité sur une aurore qui se fait encore attendre.

Nous avons l'occasion de fuir et soudain il est là qui donne des consignes. Nous devons ne pas rester trop près les uns des autres, nous déplacer ensemble mais nous tenir à bonne distance. Sans dire un mot, il nous place à bonne distance, nous prend le coude dans une main de fer. Par son sérieux absolu et son air absent dans ce moment de danger, je reconnais que c'est lui.

Un jour cet homme vous prend par le coude pour vous demander: "Dis-moi ce qui ne va pas, je ne tourne pas rond, je ne sais pas ce que c'est, tout finit mal.»

Saurez-vous lui parler sans enfreindre le respect qu'on lui doit? Quand le seul fait d'en débattre en fait un point de non-retour. L'enveloppe dans sa blessure.

\section{V}

Jouer une corrida pour soi seul : c'est la seule façon de ramener sa vie dans un éclat de dignité. C'est perdre la vie mais la rendre à elle-même, à la dignité à laquelle elle a droit.

«Il n'est plus. » Ces mots jettent un éclair froid, avec un tranchant sans pardon. Certains n'ont pas voulu être dérangés, ils ont continué leur chemin. Pour les autres, il n'y a de vérité qu'un visage découvert, ils se sont arrêtés - cette vérité est comme une pièce dans la poche de celui qui n'a plus rien.

La plupart étirent leur vie, le plus longtemps possible, comme s'ils en savaient quelque chose. Combien de temps peut-on opposer la franchise à l'hébétude? Tous ceux qui croient d'emblée posséder ce qu'ils sont, pour ensuite être ce qu'ils ont, se méfient de ceux qui ne possèdent pas, qui ne peuvent ajuster le pas et titubent. 
On peut rater sa réussite, on peut - plus rarement - réussir son échec et démontrer aux autres ce à quoi ils ont échappé. On court un plus grand risque à se donner tout entier à une seule chose, à une chose insaisissable. Il suffit d'un jour de faiblesse pour perdre pied complètement. Il est moins risqué de dépendre des autres - cela ne lâche pas tout d'un coup, cela ne cède que par morceaux. Comme on finit par s'y trouver bien, on ne se dit jamais que ce genre de vie ne vaut rien.

Ce qui aurait vraiment compté, nul n'aurait pu lui donner. Car il était de ceux pour qui les refus, les trahisons, les humiliations sont les vagues d'une fatalité qui frappe sans relâche, sous laquelle il faut rester replié, sans laisser de place à l'empoigne, pour ne pas se noyer dans le déferlement monotone.

Ceux-là relèvent la tête pour se mettre à hauteur d'horizon, parce qu'ils croient que les bonheurs d'enfant ne sont jamais étouffés. Leurs bonheurs pétillent dans leurs yeux et surpassent les souffrances que la mort s'empresse de nous épargner. Oui, il sait de quel monde le bonheur est la clef. Il se désole de ce qu'il n'a pu être, de ce qu'un petit peu de bonheur lui aurait permis d'être - et plutôt que de se désoler, il a choisi de sauter par dessus l'horizon, fouetté par une lanière de sang.

Lorsqu'il a sauté, son ombre est revenue sur lui, lui a passé à travers le corps, l'a rendu transparent. Alors les émotions, et les pensées sont à nu. Celles qu'il éprouvait, celles que nous éprouvons.

Nous avons pleuré ses rages une à une. Lloraron sus invidias una a una ${ }^{1}$.

Quevedo

\section{VI}

Tout d'abord c'est au prix d'un effort continuel que nous parvenons à rester en surface. Ensuite c'est à coup d'improvisations répétées que nous parvenons à nous créer et à nous rassembler. C'est une corrida pour soi seul, une ornière qui se creuse.

Les fous croient exister si peu qu'ils sont convaincus que les véritables fous sont les gens comme nous qui nourrissent un rêve d'immortalité avant même d'avoir commencé à exister ici même. Nous croyons en effet qu'il faut mener à terme une lutte contre la mort avant de commencer.

L'art permet à l'humanité de surmonter ses deuils. La vie n'y suffit pas. La lumière au matin n'a rien compris à l'obscurité de la nuit. L'artiste travaille à donner forme à quelque chose qui n'est pas destiné à la lumière - ce à quoi il échoue pourtant lorsqu'à vivre au plus près de sa perte, de toutes celles qui lui ont été infligées, il prend un risque qui engage sa vie même. Pour nous tous, la vie est un accident amer, certains l'auront su avant le terme, d'autres ne l'apprendront qu'en dernier lieu, avec cette différence que les noyés de l'existence s'abandonnent aux bas-fonds.

\section{VII}

Je te conjure de me maudire, de me

bénir avec des larmes de fureur

Enrage, rage de cette lumière qui meurt. Dylan Thomas

Ce qui fait du présent un marteau, et du passé une enclume, c'est le martèlement de la conscience entre les deux : combattre les rages du passé avec les pitoyables gratifications du présent. Mais il y a une rage inextinguible qui vient de ce que nous sommes chacun - dans notre façon d'exister - seuls. La souffrance est un grand fleuve qui fait son lit dans les générations et passe par ceux qu'il noie - parfois dès leur première jeunesse - avec un flot plus nombreux.

Tous les jours on s'anesthésie un peu, on se dit alors «je suis mort»sans s'étonner, avec la conviction qu'on en revient. Et puis on apprend que le seuil est mortel, parce que dans un sens ou dans l'autre quelqu'un n'est pas repassé. Pour connaître la mort, il faudrait qu'elle nous laisse revenir, sans emprisonner notre regard. Nul ne meurt à lui-même, on se croit perdu pour les autres comme nous avons perdu des êtres chers. Alors se mourir ce serait n'être mort que pour soi, ce serait y échapper, en s'engouffrant du côté d'un vide entre la vie et la mort.

\section{ÉPILOGUE}

- Vous avez vu, je suis encore loin... Mais je cherche à approcher. Parfois, il ne faut rien espérer de plus. Mais cette approche a manifestement quelque chose de sacré.

- Nous sommes toujours deux. Un vivant et un mort. Et ils sont constamment aux prises.

Bram van Velde

Quelques jours avant, il m'a appelé de son atelier. De sa fenêtre, on ne voyait que terrains vagues et excavations. Sa voix était claire, bien posée. Cette fois-ci il n'y avait pas le roulis des satellites au fond de l'océan. Il voulait aller peindre dans les Pyrénées. Et je me dis maintenant qu'il m'a appelé pour me laisser cette image de lui, buvant son petit vin blanc à flanc de montagne, peignant des paysages brûlés, fouettés par le sang, et des jeux d'arène.

Il savait combien sont douloureuses les vacillations d'une mémoire trop vive lorsque le fait que le voilà inerte s'impose à nous.

Il fait de la peinture dans les Pyrénées, la fenêtre ouverte sur un matin frais.

\section{EN MARGE DE L'AMITIÉ}

Comment présupposer chez quelqu'un une intention d'en finir, sans lui laisser le bénéfice que sa vie échappera à sa volonté ? Tout s'enchaîne alors avec une logique implacable, comme une histoire qu'on aurait écrite d'avance - ce qui a pour effet de justifier le texte, c'est-à-dire l'histoire qu'on finit par écrire effectivement, plus tard. Notre histoire sera alors faite d'observations mais aussi de bribes narratives qu'on aurait laissé «tomber » ici et là, comme s'il suffisait de les ramasser. En fait nous les aurons rassemblées selon une trajectoire inédite.

Corrida pour soi seul était d'abord un tirage modeste, qui a circulé parmi les amis de Jacques ${ }^{2}$. Certains ont mal réagi, je ne sais pourquoi, je le regrette évidemment, ce qui me laisse déstabiliser ma propre entreprise: est-ce parce que je pose l'éventualité du suicide d'entrée de jeu? En fait on devrait dire que personne ne se suicide, il y a toujours un assassin : le désespoir, le conformisme, l'indifférence, la malchance, l'abandon.

Est-ce parce que je fais mine de m'installer du côté des vivants, avec la prétention d'expliquer à qui veut l'entendre comment vivre, comment s'accrocher à la vie ? À qui veut l'entendre effectivement - mais ceux qui n'entendent pas? Sans m'interroger sur la vie à laquelle je les restitue : est-ce que je sais seulement quelle sera leur vie dorénavant? Comme si «la vie» était la même pour nous tous. Cette vie identique en tous est déjà une histoire qu'on se raconte, une histoire avec sa morale qui me permet d'encapsuler les moments de vie et de les sceller.

Dans un film de Bergman, un père note dans son carnet les étapes de l'effondrement de sa fille dans la folie. Il avoue une fascination morbide. Sa fille découvre le carnet!

Et pour raconter cette histoire, la vie sous le sceau de la vérité, on utilise les bribes par lesquelles l'autre se raconte déjà, pour les rassembler et les finaliser dans un récit particulier. L'autre n'aura été que lambeaux d'histoire, on s'en empare pour s'en donner un récit bien lisse et bien cadré, pour abriter l'illusion de notre très grande humanité dans les moments les plus critiques de la vie. On se constitue comme narrateur du récit de l'effondrement. Exhibant alors que l'on peut résister à l'effondrement, que l'on aurait encore le choix.

Alors la littérature, c'est vivre par procuration. J'étais horrifié et fasciné par le livre de Henry Miller sur ses amis.

J'étais flatté de l'importance que Jacques m'accordait. Mais aussi je prenais trop de place, sous prétexte que j'avais peur de le laisser seul. Quand on dit que tout peut arriver, c'est qu'il y a une chose que l'on craint par-dessus tout. J'étais indigné de l'attitude d'un copain de longue date que Jacques avait beaucoup aidé à Paris, qui dramatisait la crise pour se donner une excuse de rester 
à l'écart. Pourtant il me faudrait admettre à quel point je me suis protégé à ma façon : la crise prenant de l'ampleur, on peut la nommer, elle devient une entité autonome, qui dévore les personnes sous vos yeux, mais cette crise, c'est encore ces personnes !

La vie prend dimension d'événement dès qu'elle est sur le point de s'achever, dès que l'être est mis en péril. On se nourrit de ces trouées du quotidien, on s'abreuve aux effets vortex de la souffrance. J'étais en marge de cette crise, flottant en périphérie du tourbillon, libre de m'y laisser emporter quelque peu et de m'en distancer, ce qui n'a plus rien à voir, puisque le tourbillon, c'est l'individu qui n'a plus le choix entre se faire du bien et se causer du tort, entre se comporter correctement et tout mettre en déroute.

On connaît bien le rapport entre les dépendances et l'obsession de l'argent. Bien sûr, dans cette situation, celui qui «sait» vivre est celui qui a de l'argent. Du moins semble-t-il. Celui qui le flambe ne veut pas savoir vivre, il le flambe pour ne pas savoir. On croit que l'argent, c'est comme les encouragements, multiplier les exportations, assurer le soutien, créer les conditions favorables. Il n'en est rien. On se leurre sur le rôle de l'argent dans notre propre vie, à quel point ça joue un rôle de béquille morale. S'il y a un lien vital dans l'argent, il sera plus vite dépensé.

J'étais le biographe d'une personne anonyme - c'est le paradoxe, il suffit d'un biographe et vous n'êtes pas tout à fait anonyme : j'étais le monographe d'un écorché.

À sa mort, ses amis ont organisé une veillée funèbre dans l'atelier, les peintures de corrida sur les murs, les chandelles sur la table. Toute la nuit ils ont chanté, ils ont bu, ils ont fumé, David a joué du flamenco. Je n'y étais pas mais, plusieurs mois après, sa fille - il en a deux - m'a écrit un mot, pour me remercier. Elle aimait assez ce père, son deuxième, cela n'enlevait rien à tout l'amour qu'elle lui portait de le savoir complice du broyage cruel qui l'a emporté. Elle m'a dit: dans ton Corrida, tu as écrit sur Jacques comme David a joué son flamenco pour Jacques.

J'en demandais pas tant. Mais cela consolidait ce que Jacques m'avait appris, il fallait bien entendu que ma phrase se tienne debout dans l'évocation d'un individu qui se tient debout alors qu'en même temps, en dedans, il est plié de douleur. Le style, c'est l'homme, a-t-on dit. Le style, c'est l'autre homme, pourrait-on dire. Une présence de l'autre évoquée par le rythme, la scansion : il est «dans» notre rythme. J'ai découvert ce rythme, son insistance immobile lors de ma première rencontre avec les gitans, sur la proue d'un bateau entre Barcelone et Palma. La bouteille à la main, aux heures avancées de la nuit, ils se répondaient acapella, racontant leurs malheurs, laissant se dire une fierté qui ne dit qu'elle-même et qui n'a qu'elle-même - et qui se chante. Nous étions des passagers de $4^{\mathrm{e}}$ classe, ce qui nous donnait le droit de veiller sur le pont, tandis que les touristes dormaient dans le confort des cabines.

C'est de rappeler ce qu'est un cœur libre qu'on peut savoir ce qu'est la liberté. Il y a des trajectoires qui ont déjà la noblesse du poème héroïque.

Un ciel gris, une terre désolée, un froid qui fait sentir la proximité des immensités glacées, que la vie tout entière n'est qu'une poussière dans le vide. Rejoindre ainsi une pureté de l'être, sans jamais oser dire cette pureté dans laquelle on avance silencieusement et disparaît silencieusement. Cette pureté comme quelque chose de très vaste, d'invisible et d'étouffant. Oui, axphyxiant quelques instants on aura gardé son souffle, on se sera rempli de cette bouffée de vie, on aura essayé de la garder le plus longtemps.

Je retrouve aujourd'hui l'incrédulité d'avoir été tenu en si grande amitié. Ce n'est pas tant que je ne pouvais y croire, mais je ne parvenais pas à y répondre. En fait l'amitié n'attend pas de réponse, on doit en mesurer la hauteur, c'est tout. L'amitié est certes la façon d'être « vivant». Elle enseigne que, quelles que soient nos conditions matérielles, nos contraintes sociales, il y a une façon. Une façon d'être, de vivre, de se tenir. Si nombreuses et profondes nos amertumes, il y a une façon. Cette chose est si précieuse, si précieuse et rare, qu'on se console bien vite de n'avoir pas d'amis, pour peu qu'on ait déjà eu des amis. Il faut le dire, le seul fait de savoir ce qu'est l'amitié, voilà qui nous accompagne et ne nous laisse plus jamais seuls.

Voilà la nuance, rester à côté, rester à son côté. Rester à l'écart, rester de côté, de qui ? d'un ami ? de la vie? On se ménage une place dans la marge, on craint la «stase » trop violente. En x-stase à côté de l'être: x comme exclu, perdu, sacrifié. Plutôt ça peut-être, car l'être se consume, l'existence est brasier, la vie est sa lumière.

La peinture de Jacques, la poésie de mes fossés d'insomnies, tout cela travaillait à convertir l'agonie en puissance de ressourcement. Le créateur retrouve ici des gestes chamaniques, le vieux peut boire la jeunesse de ceux qui l'entourent, le malade peut boire la santé de son entourage. Il suffit d'être bien entouré. Et de boire - d'absorber en soi le flamboiement. Tandis que Neil Cassidy déroulait la pellicule d'un road movie, Jack Kerouak tapait à la machine sur un rouleau de papier un livre que le lecteur devra pourtant parcourir pages après pages.

L'art comme une brûlure laissée contre un mur. Quel mur? Celui du El Tres de Mayo de Goya.
Mais on n'atteint ce pouvoir de dissipation, de rayonnement que lorsqu'on est sur sa fin. Alors on irradie notre vie même, elle coule dans le regard qui nous observe, elle se réfugie dans le cœur qui se désole pour nous.

Comment dire tout cela on ne le saurait, la phrase est dans la vie humaine et non dans le monde.

Non dans la vie mais dans la pénombre qu'abrite le doute, dans le terrain désolé que balaie l'hésitation. Comment emprunter le langage de l'hésitation pour dire ces choses pour lesquelles l'existence semble par avance une affirmation péremptoire. Oui, l'être est péremptoire. Oui, la vie doit négocier le parcours de son effondrement. Comment emprunter aux choses des demi-affirmations pour dire ce qui se nie, s'effrite, s'étiole?

Corrida pour soi seul a été lu en public aux Journées internationales Antonin Artaud en 1993 ; j'avais demandé aux musiciens de se retirer, ce qu'ils ont fait avec dépit, je m'en excuse. Je voulais, pour lire Corrida, la solitude de l'arène, le sable brûlant qui attend le sang.

Il y a des êtres dont la vie sort du corps avant le terme, la vie terrestre n'est qu'un exosquelette. Mais ils ne savent pas où aller. Il en est d'autres dont la vie reste suspendue après leur disparition. Un corps diffus, morphique - il y a ainsi des moments de vie qui laissent leur empreinte dans l'enveloppe commune. Une sihouette brûlée dans un suaire invisible.

Il était chagrin que la vie ne veuille pas jouer, il croyait pouvoir la surprendre, échapper à ses exigences, se faufiler dans l'arrièrecour, improviser un autre équilibre - tout cela pour découvrir trop tard, beaucoup trop tard, qu'il fallait négocier au tout début, pour céder en tout. C'était son arc-boutant, le jeu avait commencé depuis longtemps. Mais il a épuisé son délai en faisant mine de discuter les règles. On voit ainsi des gens jouer avec leur chien, ils font semblant de jeter la balle, le chien s'élance, ils montrent la balle, font semblant de la jeter encore c'est le moment qu'ils préfèrent; la balle jetée, il faut attendre que le chien revienne et fasse des siennes pour nous laisser la reprendre. Ainsi la vie court autour de nous, ou plutôt nous fait courir.

J'espère survivre à quelques-uns de mes amis d'aujourd'hui. Même si pour certains on se voit peu, on se voit presque plus. Je ne manquerai pas de leur rédiger un hommage. Pourquoi attendre qu'ils ne soient plus là ? C'est absurde mais c'est comme ça, l'amitié est un devoir de témoignage.

\section{Notes}

1. Pleurèrent ses rages une à une

2. Édition à tirage limité, La Griffe d'Acier, 1990, $16 \mathrm{p}$. 